\title{
Anterior-posterior cricoid split combined with silastic T-tube stenting for subglottic stenosis in children: a single surgeon's experience
}

\author{
Yuko Bitoh $^{1} \cdot$ Yuichi Okata ${ }^{1}$ Jiro Tsugawa ${ }^{2} \cdot$ Harunori Miyauchi $^{1} \cdot$ Yosuke Aida $^{1} \cdot$ Yumiko Tachibanaki $^{1}$. \\ Yumiko Nakai ${ }^{1} \cdot$ Yuichiro Tomioka ${ }^{1}$
}

Accepted: 31 July 2018 / Published online: 10 August 2018

○) Springer-Verlag GmbH Germany, part of Springer Nature 2018

\begin{abstract}
Purpose Treatment strategies and clinical outcomes of subglottic stenosis (SGS) in children are varied due to the degree and range of stenotic lesions. The optimal surgical procedure for SGS in children is still under debate. The aim of this study was to evaluate the clinical outcomes of our anterior-posterior cricoid (APC) split technique combined with long-term T-tube stenting for grade II or III SGS in children.

Methods A retrospective chart review of children with SGS between January 2011 and December 2016 was conducted. APC split was performed via open procedure under rigid bronchoscopy. After splitting, a silastic T-tube was inserted as a stent and removed 6 months postoperatively.

Results Seven children underwent APC split during the period. All children had undergone previous tracheostomy, and APC split was performed when the children were 3-9 years old without any intraoperative complications. Median duration of T-tube stenting was 11 months, and all children were decannulated successfully. There were T-tube-related complications, including two tube-tip granulation that required intervention and one accidental T-tube removal.

Conclusion APC split is a technically simple and reproducible procedure, and it could be employed as an optimal procedure for SGS in children.
\end{abstract}

Keywords Subglottic stenosis $\cdot$ Children $\cdot$ Cricoid split $\cdot$ T-tube $\cdot$ Stent

\section{Introduction}

The surgical management of subglottic stenosis (SGS) is still challenging for pediatric surgeons and otolaryngologists. The goals of management are decannulation and normal physiologic breathing. Although there have been various procedures including anterior cricoid split (ACS) [1], laryngotracheal reconstruction (LTR) using cartilage graft [2], laser ablation, and balloon dilatations, the best treatment option for SGS in children remains controversial

Yuko Bitoh

byuko1023@gmail.com

1 Division of Pediatric Surgery, Department of Surgery, Kobe University Hospital, Kobe University Graduate School of Medicine, 7-5-2, Kusunoki-cho, Chuo-ku, Kobe 650-0017, Japan

2 Department of Pediatric Surgery, Takatsuki General Hospital, Takatsuki, Osaka, Japan because of the insufficient decannulation rate after a definitive procedure.

Thus far, we have tried to find the best treatment option for congenital and/or acquired SGS [3, 4]. As we previously reported the efficacy and feasibility of long-term T-tube stenting for severe acquired SGS [5], we considered that postoperative long-term T-tube stenting has the potential to be best treatment choice for grade II or III SGS in children. Our procedure was named simple anterior-posterior cricoid (APC) split.

The aim of our study was to describe our technique and to clarify the clinical outcome of APC split and long-term T-tube stenting for grade II or III SGS in children.

\section{Methods}

A retrospective chart review of children, who underwent laryngeal operation for SGS between January 2011 and December 2016, was performed. The information collected 
included children demographics, severity of stenosis, operative details, complications, hospital stay at surgery, duration of stenting, and the final outcomes. The SGS was graded according to the Myer-Cotton Grading System [6] by rigid bronchoscopy (HOPKINS telescope; Karl Storz, Tuttlingen, Germany). Among these children, we selected those with grade II and III as candidates of our procedure and excluded those with other airway anomalies, including tracheomalacia, congenital tracheal stenosis, and laryngomalacia. All operations were performed by a single surgeon with the informed consent of their parents.

APC split was performed as described by Cotton and Seid [1] with some modifications (Fig. 1a-c). The procedure was performed under direct and bronchoscopic vision with the child positioned on a shoulder roll. Airway control was maintained with a tracheostomy tube. A horizontal skin incision was made over the cricoid to expose the anterior surface of the larynx and upper trachea. The extent of the stenotic segment and the midline of the airway were determined by intraoperative bronchoscopic observation of a 26-gauge fine needle tip inserted into the airway. After defining the length of the stenotic segment, the midline incision was performed anteriorly in the larynx extending from the upper two tracheal rings to the lower portion of the thyroid cartilage, generally stopping just below the vocal cords. The posterior cricoid plate was divided similar to the midline, exposing
Fig. 1 APC split procedure: a the anterior midline incision; b the posterior cricoid plate is divided vertically in the midline; $\mathbf{c}$ stenting the subglottic lesion using silastic T-tube; and d overview of the larynx after surgery a

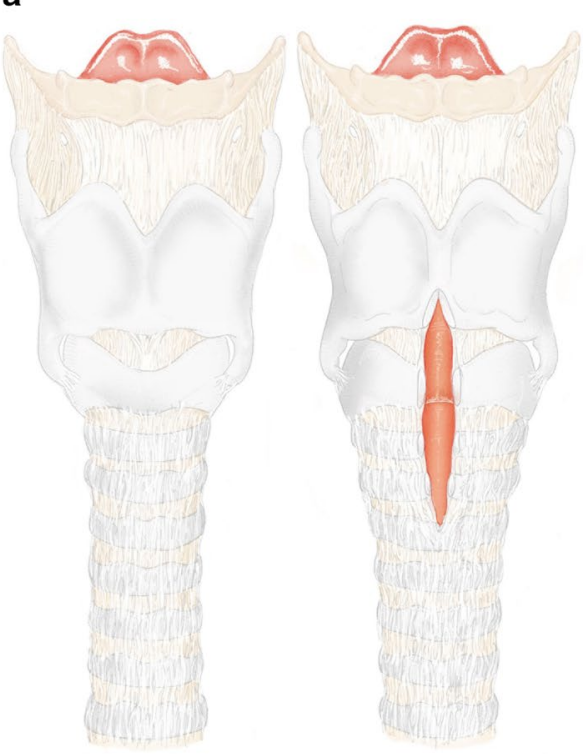

C

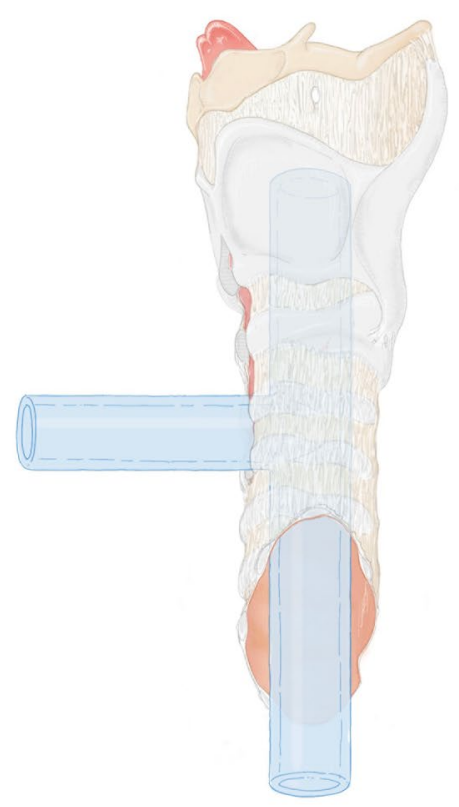

b

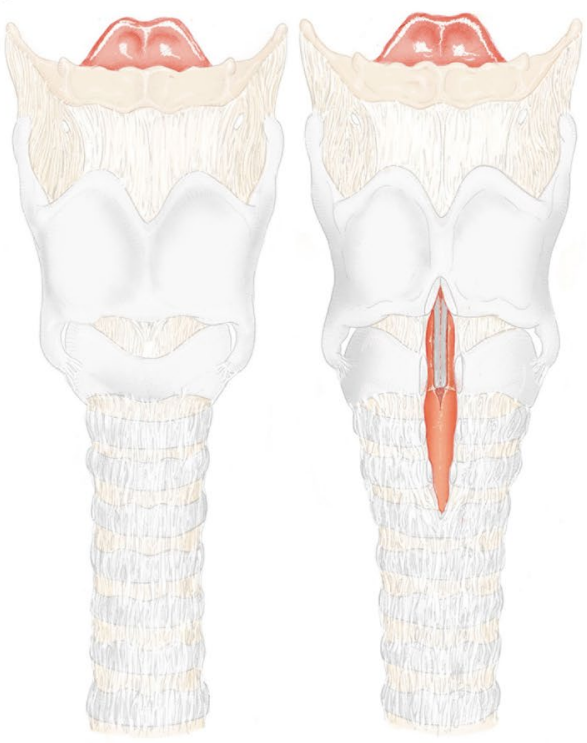

d

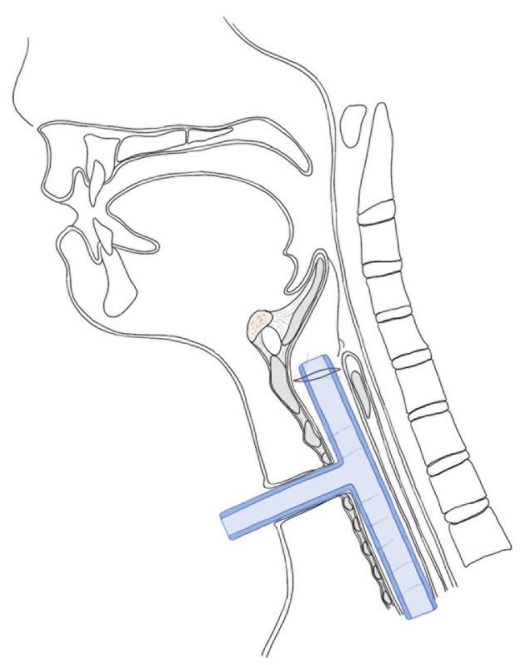


the median raphe of the cricopharyngeus and the esophageal constrictors to enlarge the subglottic lumen.

After splitting, a silastic T-tube (KOKEN, Tokyo, Japan) with 8 or 9 -mm diameter was placed as a postoperative stent. The top of the T-tube was positioned beyond the vocal cords to prevent vocal cord granulation. The limb of the tube was extended externally from the anterior neck (Fig. 1d), through the tracheostomy site, enabling the insertion of a suction tube and allowing the performance of inspiration therapy if necessary.

In the postoperative care, neither continuous intravenous sedation nor ventilator support was required. Children were permitted daily activities and usual oral intake.

The T-tube was removed endoscopically from 6 to 12 months after its placement and simultaneously endoscopic evaluations of the airway were performed. The children had no stenting at the subglottic area, and the inserted tracheostomy tube was gradually changed to a small sized tracheostomy tube for several days, and finally extubated.

\section{Results}

The demographic information of the children who underwent APCS and T-tube stenting is summarized in Table 1. Seven children were included; four of whom were male. The median age at surgery was 81 months (range 45-114 months). Four children were premature and extremely low birth weight infants; three were mature, of whom two had congenital SGS and one had acquired stenosis due to tracheal intubation for pulmonary injury because of a traffic accident. One child was initially diagnosed with esophageal atresia (Gross type C), and intubation of an adequate size of tracheal tube through the cricoid plate during a definitive operation for the esophagus at the first day of life failed. Tracheostomy was performed on the 12th day.
Moreover, one child had grade II stenosis, and six had grade III stenosis during the APC split. All children had preoperative tracheostomy at the time of diagnosis, and none of them had been previously treated with other airway surgeries.

The average operative time was 130 min (range 92-173 min), and average blood loss was $9.4 \mathrm{~g}$ (range 2-28 g). There was no intraoperative complication, and none of the children required blood transfusion. The children were transferred to the intensive care unit or high care unit postoperatively without intravenous sedation. Postoperative outcomes are shown in Table 2. All children started oral intake on postoperative day 1 , with coughing during meals induced by aspiration due to the T-tube stenting, which gradually improved later; none had pneumonia. One child had wound bleeding that required hemostasis on postoperative day 2. Median duration of T-tube stenting was 11 months (range 5-13 months) and all children were decannulated successfully. We encountered T-tuberelated complications, including tube-tip granulation that required intervention and accidental T-tube removal. Three children had tube-tip granulations at the supraglottic area, two of whom were managed by laser ablations. The accidental removal of the T-tube occurred in one female child 5 months postoperatively while she was playing with her sister at home. The child was immediately brought to the hospital and a T-tube of the same size was inserted to the tracheostomy site under general anesthesia and stability was checked using a bronchoscopy.

The median follow-up period after APC split was 18 months (range 8-72 months). Outcomes were assessed on the basis of symptomatic improvement, airway assessment, and tracheostomy decannulation. None of the children demonstrated signs of dyspnea. On the 6 months after surgery, all bronchoscopic evaluations showed grade I stenosis (Fig. 2).
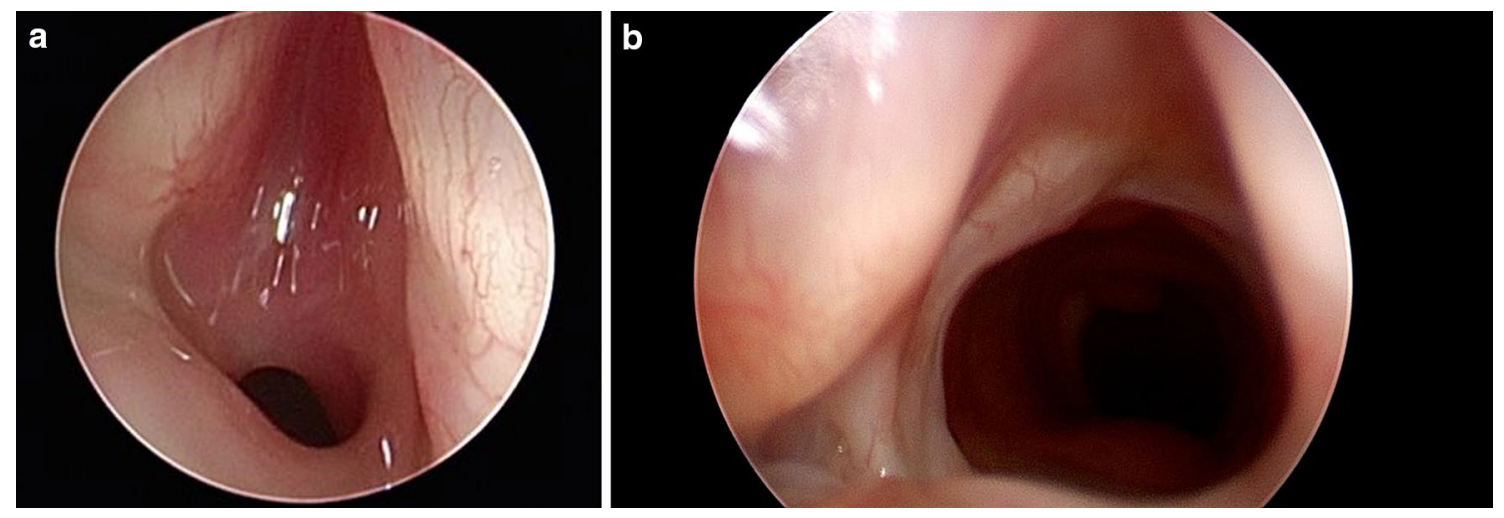

Fig. 2 Endoscopic results of APC split for grade III subglottic stenosis in case 4. a Preoperative view: the grade III subglottic stenosis. b Postoperative view at 4 months after removal of stent: large, round-shaped subglottic lumen 


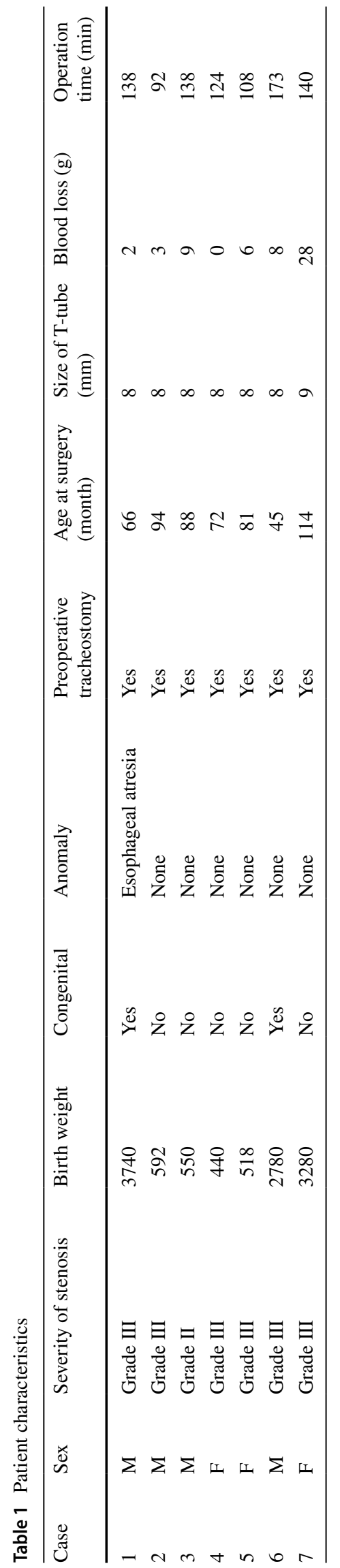

\section{Discussion}

We present the first study on the efficacy of combined therapy of the anterior-posterior cricoid split and longterm T-tube stenting (APC split). The present study demonstrated $100 \%$ decannulation rate of ACS with T-tube stenting in children with grade II or III acquired SGS. All children had no severe restenosis after the procedure, which was estimated as grade I stenosis. Oral intake was started from postoperative day 1 , and the children had no blood transfusion and postoperative pneumonitis. One child had continuous bleeding from the wound site postoperatively and was treated by hemostasis.

Compared with the historical data, our procedure revealed a high decannulation rate [7]. This high success rate of overall decannulation in these children can be explained by several advantages. Firstly, we limited the indication of the procedure for grades II-III. Secondly, as our procedure is more simple touch to the lesion and less invasive than LTR using cartilage graft, excess granulation and/or inflammation might occur at the subglottis area after the operation. Lastly, a long-term stent using a silastic T-tube might produce a rigid framework at the split site and enlarge the subglottic lumen.

In the past, we selected the LTR using a costal cartilage graft for pediatric SGS. Our clinical impression was that LTR might not be reproducible and was technically complex to perform. In spite of the effort to precisely perform the procedure, deviation of the graft might occur, and the procedure sometimes might induce distorted shapes of the subglottic lumen after the operation, leading to restenosis. Although it has been considered that the cartilage grafting promotes earlier healing of the lesion, LTR using cartilage graft does not always result in a high decannulation rate.

Our previous report [5] described that T-tube stenting had the tendency to create a completely round-shaped lumen. A T-tube is made of soft silastic material that offers moderate intraluminal support against collapse. Moreover, the quality of life of the patients during stenting could be maintained because home care is easy and their respiratory status is good because of airway patency. Therefore, we decided to adapt the APC split and used the T-tube as a postoperative stent, and to keep and stabilize the enlarged subglottic lumen by long-term stenting for grade II and III SGS in children.

Recently, balloon dilatation is preferred to manage SGS as the minimally invasive treatment $[8,9]$. However, the mode of destruction cannot be anticipated by this procedure, despite of the formation of cutting edge. It is supported by the result from balloon dilatation for congenital tracheal stenosis due to complete tracheal ring $[10,11]$. Additionally, the damage in another site, including the 
Table 2 Postoperative outcomes

\begin{tabular}{llllll}
\hline Case & $\begin{array}{l}\text { Postoperative } \\
\text { complication }\end{array}$ & $\begin{array}{l}\text { Hospital stay at } \\
\text { surgery (day) }\end{array}$ & $\begin{array}{l}\text { Duration of stent- } \\
\text { ing (month) }\end{array}$ & $\begin{array}{l}\text { Successful } \\
\text { decannulation }\end{array}$ & $\begin{array}{l}\text { Respiratory distress } \\
\text { after removal of } \\
\text { stent }\end{array}$ \\
\hline 1 & $\begin{array}{l}\text { Oozing from } \\
\text { wound }\end{array}$ & 15 & 13 & Yes & None \\
2 & $\mathrm{~N}$ & 15 & 11 & Yes & None \\
3 & $\mathrm{~N}$ & 26 & 7 & Yes & None \\
4 & $\mathrm{~N}$ & 26 & 12 & Yes & None \\
5 & $\mathrm{~N}$ & 10 & 11 & Yes & None \\
6 & $\mathrm{~N}$ & 16 & 13 & Yes & None \\
7 & $\mathrm{~N}$ & 24 & 5 & Yes & None \\
\hline
\end{tabular}

vocal cords, is concerning. Therefore, we thought that open surgery including APC split has better results compared to balloon dilatation.

The complications of the T-tube stenting were postoperative aspiration, granulation at the top of the T-tube that required laser ablation, and unplanned decannulation of the T-tube that required reinsertion. We found that aspiration improved gradually in the children because they got used to swallowing their meals. However, it is necessary that the T-tube is not too long to allow normal swallowing. In terms of granulation and inflammation, children were administered budesonide 2-4 times per day via inhalation for postoperative management, according to a previous report $[12,13]$, which was effective. The unplanned decannulation occurred in the children's daily activities. It is necessary for parents or caregivers to always ensure that the T-tube is untouched and must not be hooked carefully.

The appropriate length of the stenting period using T-tube is still unknown [14]. The stents were removed in the children after 6 months postoperatively, but we supposed that it might be shortened.

Our study was subject to several limitations. First, this was a retrospective case series study with a small sample size. Second, biases could have been caused by the diagnostic decision and surgical judgment during this study period. Accordingly, large, prospective, and multicenter studies are needed to validate our findings in the future. Despite these limitations, we believe that our technique is simple and reproducible, and could be one of the best options for grade II or III SGS in children.

\section{Conclusion}

We report high rates of decannulation for congenital and acquired SGS in children using APC split and long-term T-tube stenting. APC split with T-tube stenting is a simple and reproducible procedure, and it could be one of the optimal procedures for SGS in children. Further research is needed to better identify the characteristics of the ideal candidates for APC split with T-tube stenting.

\section{Compliance with ethical standards}

Conflict of interest The authors declare that they have no conflict of interest.

Ethical approval All procedures performed in studies involving human participants were in accordance with the ethical standards of the institutional and/or national research committee and with the 1964 Helsinki declaration and its later amendments or comparable ethical standards. The Ethics Committee considered this study as case series and confirmed that ethics approval was waived. For this type of study formal consent are not required.

\section{References}

1. Cotton RT, Seid AB (1980) Management of the extubation problem in the premature child: anterior cricoid split as an alternative to tracheostomy. Ann Otol Rhinol Laryngol 89:508-511

2. Cotton RT, Evans JN (1981) Laryngotracheal reconstruction in children. Five-year follow-up. Ann Otol Rhinol Laryngol 90:516-520

3. Okamoto M, Nishijima E, Yokoi A, Nakao M, Bitoh Y, Arai H (2012) Strategy for surgical treatment of congenital subglottic stenosis in children. Pediatr Surg Int 28:1115-1118

4. Morita K, Yokoi A, Bitoh Y, Fukuzawa H, Okata Y, Iwade T, Endo K, Takemoto J, Tamaki A, Maeda K (2015) Severe acquired subglottic stenosis in children: analysis of clinical features and surgical outcomes based on the range of stenosis. Pediatr Surg Int 31:943-947

5. Zaima A, Bitoh Y, Morita K, Tsugawa J, Ishii T, Satoh S, Nishijima E (2010) Long-term T-tube stenting as definitive treatment of severe acquired subglottic stenosis in children. J Pediatr Surg 45:996-999

6. Myer CM, O’Connor DM, Cotton RT (1994) Proposed grading system for subglottic stenosis based on endotracheal tube sizes. Ann Otol Rhinol Laryngol 103:319-323

7. Jefferson ND, Cohen AP, Rutter MJ (2016) Subglottic stenosis. Semin Pediatr Surg 25:138-143

8. Hebra A, Powell DD, Smith CD, Othersen HB Jr (1991) Balloon tracheoplasty in children: results of a 15-year experience. J Pediatr Surg 26:957-961 
9. Bent JP, Shah MB, Nord R, Parikh SR (2010) Balloon dilation for recurrent stenosis after pediatric laryngotracheoplasty. Ann Otol Rhinol Laryngol 119:619-627

10. Maeda K (2017) Pediatric airway surgery. Pediatr Surg Int 33:435-443

11. Ono S, Maeda K, Baba K, Usui Y, Tsuji Y, Kawahara I, Fukuta A, Sekine S (2014) Balloon tracheoplasty as initial treatment for neonates with symptomatic congenital tracheal stenosis. Pediatr Surg Int 30:957-960
12. Roh HJ, Goh EK, Chon KM et al (1999) Topical inhalant steroid (budesonide, pulmicort nasal) therapy in intubation granuloma. J Laryngol Otol 113:427-432

13. Brigger MT, Hartnick CJ (2009) Laryngotracheal reconstruction. Oper Tech Otolaryngol 20:229-235

14. Smith DF, Alarcon A, Jefferson ND, Tabangin ME, Rutter MJ, Cotton RT, Hart CK (2018) Short- versus long-term stenting in children with subglottic stenosis undergoing laryngotracheal reconstruction. Otolaryngol Head Neck Surg 158:375-380 